\title{
Drug Resistance Patterns of Salmonellae Enterica Serotype Typhi and Paratyphi at a Tertiary Care Hospital of Sindh, Pakistan
}

\author{
Haji Khan Khoharo ${ }^{1}$ and Iqbal Ahmed Memon ${ }^{2}$ \\ ${ }^{1}$ Faculty of Medicine \& Allied Medical Sciences, Isra University Hyderabad, Sindh, Pakistan \\ ${ }^{2}$ Department of Medicine, Liaquat University of Medical \& Health Sciences \\ Jamshoro, Sindh, Pakistan
}

Correspondence should be addressed to: Haji Khan Khoharo; drhajikhan786@gmail.com

Received Date: 29 October 2013; Accepted Date: 22 January 2014; Published Date: 28 February 2014

Academic Editor: Sushant Sahastrabuddhe

Copyright @ 2014 Haji Khan Khoharo and Iqbal Ahmed Memon. Distributed under Creative Commons CC-BY 3.0

\begin{abstract}
The present study was conducted to evaluate drug resistance patterns of salmonella enterica serotype typhi and paratyphi in enteric fever.
\end{abstract}

This study is an observational study.

The place of this study is the Department of Medicine, Liaquat University of Medical and Health Sciences Hospital and the duration from June 2012 to March 2013.

A sample of sixty seven patients was selected according to clinical and laboratory criteria. Venous blood samples of 5-10 ml were collected from antecubital veins and inoculated on blood culture bottles. Subcultures were also performed. Drug sensitivity patterns of salmonellae were tested according to standard method. Data was analyzed on SPSS version 21.0. using appropriate statistical testing.

Of sixty seven, $41(61.1 \%)$ were male and $26(38.8 \%)$ female. The male to female ratio was 1.57:1. The mean age of subjects was $43 \pm 7.5$ years. Salmonella enterica serotype typhi was isolated from $60(89.5 \%)$, salmonellae enterica serotype typhi and salmonellae enterica serotype paratyphi $A$ and $B$ were found in $3(4.4 \%)$ and $4(5.9 \%)$ respectively. The Widal test yielded antibody $O$ titers of $\geq 1: 160$ and $<1: 160$ in $57(85 \%)$ and $10(14.9 \%)$ respectively. The typhidot test was positive in $65(97.5 \%)$ of patients. The multidrug resistance to first line drugs was observed in $53(79.1 \%)$. Least drug resistance was found for cefixime $2(2.7 \%)$ and highest for the chloramphenicol $56(83.6 \%)$.

The present study observed drug resistant and multi-drug resistant S,. enterica serotype typhi and S,. enterica serotype paratyphi A and B.

Keywords: Drug sensitivity Enteric fever Salmonellae typhi/paratyphi. 


\section{Introduction}

Enteric fever is commonly known as typhoid fever. Salmonella enterica serotype typhi and salmonellae enterica serotype paratyphi are most common etiological agents of enteric fever. The term typhoid fever applies when serotype typhi is causative agent. Salmonellae are transmitted by contaminated food, milk, water or drinks. Clinical manifestations vary from person to person. The incubation period varies from 5 to 14 days (Schwartz et al 2012) (Ochiai et al 2008). Pakistan is hyper-endemic area for enteric fever. Its incidence was 412 per million in children of 5-15 years (Ochiai et al 2008). The isolation of Salmonellae enterica serotype typhi/paratyphi from blood, bone marrow and urine is necessary for definitive diagnosis; however bone marrow cultures are regarded as "gold standard method". The Widal and typhidot tests are alternative serology methods for diagnosis (Behrmann et al 2008). The drug resistance of salmonellae was rare prior to 1980s. The treatment of choice was chloramphenicol, but emergence of resistance in mid 1980s, created therapeutic problems particularly in countries where enteric fever is endemic. Then the ciprofloxacin became drug of choice for typhoid (Somily 2010). The S. enterica serotype typhi resistant to chloramphenicol, ampicillin, and cotrimoxazole were reported from South America, Africa, India and Saudi Arabia (Somily 2010). Simultaneously, Southern Vietnam reported multi-drug resistance isolates in early 1990s (Somily 2010). Shortly after, isolates of $S$. enterica serotype typhi resistant to quinolone were reported from same geographical areas with resistance to first line drugs. This was a major threat for treatment of typhoid fever. Isolates of $S$. enterica serotype typhi resistant to nalidixic acid and ciprofloxacin increased to $23 \%$ in United Kingdom in 1999s. Somily (2010) reported drug resistant isolates of $S$. enterica serotype typhi in patient's returing from travel to Asia (Somily 2010). The quinolones and $3^{\text {rd }}$ generation cephalosporins are reported of having successful cure rates of $82-97 \%$ and $100 \%$ respectively (Hazir 2010). But a number of resistance mechanisms to quinolone in certain parts of the world led to decreased susceptibility of $S$. enterica serotype typhi to these drugs also (Somily 2010). Like many tropical areas, enteric fever is hyper-endemic in Pakistan. It is major public health problem and a cause of mortality in Sindh. The present study was aimed to have insight into current trends of drug sensitivity and resistance patterns of S. enterica serotype typhi and salmonellae enterica serotype paratyphi in Sindh province.

\section{Methodology}

Sixty seven adult patients with a clinical diagnosis suggestive of enteric fever, admitted at Department of Medicine, Liaquat University of Medical and Health Sciences Hospital from June 2012 to March 2013, were enrolled in the study. The clinical history of symptoms and drugs was sought from the patients. Enteric fever was defined according to gold standard method of isolation of $S$. enterica serotype typhi and salmonellae enterica serotype paratyphi A and B on blood culture. (Konemann 1997). Venous blood samples were collected from febrile patient whether taking or not an antibiotic therapy. Complete blood counts, Widal test, typhoid test and blood cultures were carried out according to standard methods by hematologist. Bottles containing $50 \mathrm{ml}$ of brain heart infusion broth were inoculated with blood for culture. The samples were processed according to standard recommended techniques for blood culturing. (Konemann 1997). The blood cultures were incubated at $37^{\circ} \mathrm{C}$ for seven days and bacterial growth was observed on daily basis. Subcultures were performed on $1^{\text {st }}, 3^{\text {rd }}, 5^{\text {th }}$ and $7^{\text {th }}$ day of incubation on 5\% sheep blood agar and Mac Conkey medium (Kumar 2008) Nonlactose fermenting colonies were screened by biochemical reactions, and further identified by serology. Antibiotic sensitivity and resistance of microorganisms were tested according to standardized KirbyBauer disc diffusion method. (Bauer 1966). Antimicrobial susceptibility patterns were determined using commercial antimicrobial disks (Oxoid, Basingstoke, UK). Antimicrobial susceptibility testing 
against chloramphenicol (30ug), cotrimoxazole (25ug), ampicillin (10ug), amoxicillin (10ug), amoxi-clav (20/10ug), ciprofloxacin (5 ug), cefotaxime (10ug), ceftriaxone $(10 \mathrm{ug})$, levofloxacin $(5 \mathrm{ug})$, and cefixime (10ug) performed by Clinical Laboratory Standard Institute (CLSI 2010). The study was approved by the ethics committee of institute. Data was analyzed on SPSS version 21.0. using the chi-square and student's t-tests for categorical and continuous variables respectively.

\section{Results}

The mean age of subjects was $43 \pm 7.5$ years. Of sixty seven adult subjects, $41(61.1 \%)$ were male and 26 (38.8\%) were female. $(p=0.001)$ The male to female ratio was 1.57:1. The demographic characteristics of patients and clinical symptoms and signs are summarized in table I.

Clinical Features: The most frequent clinical symptoms and signs were fever, chills headache and anorexia. The bloody diarrhea was also observed. The hepatosplenomegaly were noted. The

Antimicrobial drugs taken as self medication was noted in twenty nine $(43.2 \%)$ of patients; and it was also noted frequency and \% of clinical symptoms and signs are shown in table I. Out 67 subjects, leukocytosis and leukopenia were noted in 31 (46.2\%) and 29 (43\%) respectively.

Isolation Rate: The S.enterica serotype typhi, S. enterica serotype typhi/paratyphi and S.enterica serotype paratyphi $A$ and $B$ was isolated from $60(89.5 \%), 3(4.4 \%)$ and $4(5.9 \%)$ respectively $(\mathrm{p}=0.001)$.

Serological Tests: Typhoid test was positive in $65(97.5 \%)$ of patients. Antibody $O$ titers of $\geq 1: 160$ and $<1: 160$ were detected in 57 (85\%) and 10 (14.9\%) of patients respectively.

Drug Resistance Patterns: The antibiotic sensitivity and resistance patterns are shown in table II. The multidrug resistance to first line drugs was observed in 53 (79.1\%). The highest and lowest drug sensitivity were noted for cefixime and chloramphenicol in 65 (97.3\%) and 11 (16.4\%) of patients respectively. Least drug resistance was found for cefixime $2(2.7 \%)$ and highest for chloramphenicol 56 (83.6\%).

that the patients were running fever and were found blood cultures positive in $59.7 \%$.

Table I. Demographic Data and Clinical Features of Study Population (N=67)

\begin{tabular}{|l|l|l|}
\hline & No. of cases & \% \\
\hline Male (M) & 41 & 61.1 \\
\hline Female (F) & 26 & 38.8 \\
\hline Fever & 67 & 100 \\
\hline Chills & 49 & 73.1 \\
\hline Headache & 53 & 79.1 \\
\hline Sore throat & 27 & 40.2 \\
\hline Coated tongue & 32 & 47.7 \\
\hline Anorexia & 65 & 97 \\
\hline Vomiting & 21 & 31.3 \\
\hline Bloody diarrhoea & 11 & 16.4 \\
\hline Rose spots & 13 & 19.4 \\
\hline Meningism & 21 & 31.3 \\
\hline Hepatomegaly & 05 & 07.4 \\
\hline Splenomegaly & 19 & 28.3 \\
\hline Respiratory symptoms & 31 & 46.2 \\
\hline
\end{tabular}


Table II. Antibiotic Sensitivity and Resistance Patterns of Isolated Salmonellae

\begin{tabular}{|c|c|c|}
\hline Drug & \multicolumn{2}{|c|}{ Drug Sensitivity patterns (\%) } \\
\hline & Sensitive (\%) & Resistance (\%) \\
\hline Chloramphenicol (30ug) & $11(16.4 \%)$ & $56(83.5 \%)$ \\
\hline Co-trimoxazole (25ug) & $13(19.4 \%)$ & $54(80.5 \%)$ \\
\hline Ampicillin (10ug) & $12(17.9 \%)$ & $55(82.0 \%)$ \\
\hline Amoxicillin (10ug) & $17(25.3 \%)$ & $50(74.6 \%)$ \\
\hline Amoxi-Clav (20/10ug) & $26(38.8 \%)$ & $41(61.1 \%)$ \\
\hline Ciprofloxacin $(5 u g)$ & $23(34.3 \%)$ & $44(65.6 \%)$ \\
\hline Cefotaxime (10ug) & $33(49.2 \%)$ & $34(50.7 \%)$ \\
\hline Ceftriaxone $(10 \mathrm{ug})$ & $51(76.1 \%)$ & $16(23.8 \%)$ \\
\hline Levofloxacin (5ug) & $61(91.0 \%)$ & $6(8.9 \%)$ \\
\hline Cefixime(10ug) & $65(97.3 \%)$ & $2(2.9 \%)$ \\
\hline
\end{tabular}

\section{Discussion}

The multi-drug resistance defined as resistance to first line drugs i.e. chloramphenicol, cotrimoxazole, and ampicillin, was noted in $79.1 \%$ of $S$. enterica serotype typhi/paratyphi in present study. The results are comparable to previous studies which have reported 80-90\% (Hatta 2008) and100\% (Misra 2005). The increased drug resistance results from the exploitation of drug use by chemist, druggist, quacks, and paramedics and more over by patients himself as self medication. The access to drugs is very easy in our country because drug prescription and sale is uncontrolled by law due to prevalent political, social and other issues (Siddiqui et al 2006) (Abdullah 2012). The regulations of drug prescription and sale are not obliged by pharmacists. This results in injudicious administration and sub-optimal drug dosaging which have contributed much to the reduced susceptibility and emergence of multi drug resistant $S$. enterica serotype typhi/paratyphi, not only to the first line drugs but also to the cephalosporins, quinolones, and macrolides. (Siddiqui et al 2006) (Abdullah 2012).

One study from Indonesia reported that up to $90 \%$ of enteric fever patients were receiving antimicrobials as self medication at home and bed rest; (Hatta 2008) other patients, those with more severe disease and those treated at home who then developed persisting complaints, presented at the health care centers and hospitals
(Tjiandi 2003). The similar conditions of drug usage are prevailing in our society. A recent study has reported overall multi drug resistance in Salmonellae in enteric fever of $62.64 \%$ (Abdullah 2012) which is low but yet comparable to our present study. The recently reported amoxicillin resistance of $96.4 \%$ is higher as we found $74.6 \%$, and co-trimoxazole resistance of $29.91 \%$ is very low as compared to $80.5 \%$ in our present study (Abdullah 2012). We found cefixime, levofloxacin and ceftriaxone sensitivity of $97.3 \%, 91 \%$ and $76.1 \%$ respectively. Our findings are comparable to previous studies (Siddiqui et al 2006) (Kumar et al 2008) (Abdullah 2012). Our multi-drug resistance finding of $79.1 \%$ is comparable to previous reports from Nigeria of $80 \%$ (Akineymi et al 2007) and Pakistan(Hassan et al 2008) (Butt et al 2005). Possibly due to socio-economic constraints and indiscriminate drug use, the prevalence of quinolone resistance has gradually increased (Mubeena et al. 2000) (Fangtham et al 2008). The first case of fluoroquinolones treatment failure was reported in Pakistan in 1993 (Butt et al 2005). We found drug resistance salmonellae to ciprofloxacin and levofloxacin of $65.6 \%$ and $8.9 \%$ respectively. A study from Agha Khan University reported multidrug resistant $S$. enterica serotype typhi in $66 \%$ of children from Karachi (Khan et al 2012). Similarly a study from India reported that out of 107 salmonellae isolates, 94 (87.8\%) were nalidixic acid-resistant (NAR) Salmonella and 36 were resistant to azithromycin. Among the 57 treatment 
histories, 19 (33\%) patients had documented clinical non-response to azithromycin which required change of therapy (Rai et al 2012). This is seemingly an alarming situation showing development of drug resistant salmonellae against macrolides, quinolone and fluoroquinolones which may increase the resistance in near future. Fluoroquinolones are under strict prescription of clinicians in advanced countries (Fangtham et al 2008) but freely available in developing countries like Pakistan (Abdullah 2012). In our study the crucial finding is that the twenty nine $(43.2 \%)$ of patients taking antibiotic therapy yielded positive blood cultures in $59.7 \%$ of them, this reveals the existence of drug resistant salmonellae in the community. The easy access of public to the prescription drugs is an illegal practice and must be controlled and this may help to prevent widespread drug resistant salmonellae in future.

\section{Conclusion}

The present study observed drug resistant and multi-drug resistant $S$, enterica serotype typhi/paratyphi $A \& B$ in majority of enteric fever patients. The increasing drug resistant salmonellae must be controlled to prevent epidemics of enteric fever within community.

\section{References}

Abdullah, F. E., Haider, F., Fatima, K., Irfan, S. \& Iqbal, M. S. (2012). "Enteric Fever in Karachi: Current Antibiotic Susceptibility of Salmonellae Isolates," Journal of College of Physicians and Surgeon Pakistan, 22 (3) 147-50.

Akinyemi, K. O., Smith, S. I., Oyefolu, A. O., Fasure, K. A., Coker, A. \& Akitoye, O. (2007). "Trends of Multiple Drug Resistance in Salmonella Enterica Serovar Typhi in Lagos, Nigeria," East and Central African Journal of Surgery, 12 83-8.

Bauer, A. W., Kirby, W. M., Sherris, J. C. \& Turck, M. (1996). "Antibiotic Susceptibility Testing by a Standardized Single Disk Method," American Journal of Clinical Pathology, 45(4) 493-496.
Behrmann, R. E., Kliegman, R. \& Neslon, W. E. (1996). Nelsons Textbook of Paediatrics. 15th Ed. Philadelphia: WB Saunders.

Butt, T., Ahmed, R. N., Salman, M. \& Kazmi, S. Y. (2005). "Changing Trends in Drug Resistance among Typhoid Salmonellae in Rawalpindi, $\quad$ Pakistan," Eastern Mediterranean Health Journal, 11 103844.

Clinical and Laboratory Standard Institute (CLSI 2010). 'Performance Standards for Antimicrobial Susceptibility Testing; Twentieth Informational Supplement,' M100 S20: 30.

Fangtham, M. \& Wilde, H. (2008). "Emergence of Salmonella Paratyphi a as a Major Cause of Enteric Fever: Need for Early Detection, Preventive Measures and Effective Vaccines," Journal of Travel Medicine, 15 (5) 344-50.

Hasan, R., Zafar, A., Abbas, Z., Mahraj, V., Malik, F. \& Zaidi, A. (2008). "Antibiotic Resistance among Salmonella Enteric Serovar Typhi and Paratyphi a in Pakistan (2001-2006)," The Journal of Infection in Developing Countries, 2 (4) 289-94.

Hatta, M. \& Ratanwati, N. (2008). "Enteric Fever in Endemic Areas of Indonesia: An Increasing Problem of Resistance," The Journal of Infection in Developing Countries, 2 (4) 279-282.

Hazir, T., Qazi, S. A., Abbas, K. A. \& Khan, M. A. (2002). "Therapeutic Re-Appraisal of Multiple Drug Resistant Salmonella Typhi (MDRST) In Pakistani Children," Journal of Pakistan Medical Association, 52 (3) 123124.

Khan, M. I., Soofi, S. B., Ochiai, R. L., Khan, M. J., Sahito, S. M., Habib, M. A., et al. (2012). "Epidemiology, Clinical Presentation and Patterns of Drug Resistance of Salmonella Typhi in Karachi, Pakistan," The Journal of Infection in Developing Countries, 6 (10) 704-14.

Koneman, E. W., Allen, S. D., Janda, W. M., Schreckenberger, P. \& Winn, W. C. (1997). Antimicrobial Resistance, Color Atlas and Text Book of Diagnostic Microbiology, Fifth 
Edition, USA (Philadelphia), LippincottRaven Publishers.

Kumar, S., Rizvi, M. \& Berry, N. (2008). "Rising Prevalence of Enteric Fever Due to Multidrug-Resistant Salmonella: An Epidemiological Study," Journal of Medical Microbiology, 57 1247-1250.

Misra, R. N., Bawa, K. S., Magu, S. K., Bhandari, S., Nagendra, A. \& Menon, P. K. (2005). "Outbreak of Multi-Drug Resistant Salmonella Typhi Enteric Fever in Mumbai Garrison," Medical Journal Armed Forces India, 61 148-150.

Mubeena, R. S., Saleem, A. K. \& Ameena, R. S. (2006). 'Prevalence of Enteric Fever in Karachi,' Infectious Diseases Journal of Pakistan, 15 103-5.

Ochiai, R. L., Acosta, C. J., DanovaroHolliday, M. C., Baiqing, D., Bhattacharya, S. K. \& Agtini, M. D. (2008). "A Study of Typhoid Fever in Five Asian Countries: Disease Burden and Implications for Control," Bulletin of the World Health Organization, 86 260-68.

Rai, S., Jain, S., Prasad, K. N., Ghoshal, U. \& Dhole, T. N. (2012). "Rationale of Azithromycin Prescribing Practives for Enteric Fever in India," Indian Journal Medical Microbiology, 30 (1) 30-3.
Schwartz, B. S. \& Chambers, H. F. (2012). Enteric Fever. In: Mc-Phee, S.J., Papadakis, M.A. (Editors). Current Medical Diagnosis and Treatment, 51st Ed. New York: Mc Graw-Hill, 1405-1407.

Siddiqui, F. J., Rabbani, F., Hasan, R., Nizami, S. Q. \& Bhutta, Z. A. (2006). "Typhoid Fever in Children: Some Epidemiological Considerations from Karachi, Pakistan," The Journal of Infection in Developing Countries, 10 (3) 215-22.

Somily, A. M. (2010). "An Important Enteric Fever Caused by a Quinolone-Resistant Salmonella Typhi," Annals of Saudi Medicine, 30(4) 313-316.

Tjaniadi, P., Lesmana, M., Subekti, D., Machpud, N., Komalarini, S., Santoso, W., et al. (2003). "Antimicrobial Resistance of Bacterial Pathogens Associated with Diarrheal Patients in Indonesia," The American Journal of Tropical Medicine and Hygiene, 68 (6) 666-70. 